\title{
Creación de ambientes de aprendizaje en la enseñanza de polígonos; una experiencia de aula desde la educación matemática crítica ${ }^{1}$
}

\author{
Creating learning environments in teaching polygons from a \\ classroom experience critical mathematics education
}

\section{Criar ambientes de aprendizagem em polígonos de ensino a partir de uma experiência de sala de aula da educação matemática crítica}

Recibido: mayo de 2013

Aceptado: agosto de 2013
Julián David Martínez Hernández²

Juan Manuel Alvarez ${ }^{3}$

\section{Resumen}

Día a día observamos como las problemáticas sociales inciden en el aula y como el aula se adapta de diversas maneras a dichas problemáticas. En los últimos años una fuerte corriente de educación matemática propone la inclusión de dichas problemáticas al trabajo en el aula. Este documento se propone mostrar una experiencia de aula donde colaborativamente se crearon ambientes de aprendizaje que respondieran a las problemáticas de la comunidad y se plantea una posible secuencia de actividades para la enseñanza de geometría y espacialidad en quinto grado de educación básica.

Palabras clave: Educación matemática crítica; Ambientes de aprendizaje; Matemáticas Escolares; Geometría; Formas geométricas.

\begin{abstract}
Day by day we see how social problems affect the classroom and the classroom is adapted in various ways to these issues. In recent years a strong current of mathematics education proposes the inclusion of these issues in the classroom work. This paper aims to show a classroom experience where collaborative learning environments were created to respond to the problems of the community and is a feasible sequence of activities for teaching geometry and spatiality in the fifth grade of primary education.
\end{abstract}

Keywords: critical mathematics education, learning environments, Mathematics Education, Geometry, Geometric Shapes.

\section{Resumo}

Dia a dia, vemos como os problemas sociais afetam a sala de aula e na sala de aula é adaptada de várias maneiras para estas questões. Nos últimos anos, uma forte corrente de educação matemática propõe a inclusão dessas

1 Artículo de Investigación.

2 Universidad Distrital Francisco José de Caldas. Bogotá, Colombia. Contacto: jdmartinezh@unal.edu.co

3 Universidad Distrital Francisco José de Caldas. Bogotá, Colombia. Contacto: juanmanue1943@hotmail.com 
questões no trabalho em sala de aula. Este trabalho tem como objetivo mostrar a experiência de sala de aula onde os ambientes de aprendizagem colaborativa foram criados para responder aos problemas da comunidade e é uma sequência viável de atividades para o ensino de geometria e espacialidade na quinta série do ensino fundamental.

Palavras-chave: educação matemática crítica, ambientes de aprendizagem, Educação Matemática, Geometria, formas geométricas.

\section{Contextualización}

Problemas sociales existen en todas las comunidades, de hecho la necesidad de resolverlos es el incentivo para construir y reformar los conocimientos o las prácticas cotidianas; es la razón de mejorar día a día. Con este proyecto se busca responder a la pregunta: ¿Cómo enseñar polígonos partiendo del contexto social del alumno? Nuestra motivación es innovar, transformar la sociedad y la educación para que las problemáticas que se observan y plantean puedan ser corregidas.

La presente experiencia en el aula se propuso la creación de ambientes de aprendizaje que respondieran a la necesidad de presentar un cambio social en una comunidad específica, se desarrolló en una institución educativa de carácter privado ubicada en la localidad No 8 de Bogotá (Kennedy) en una población de estudiantes de grado quinto que habitan en el suroccidente bogotano. El curso mostró resultados positivos en la implementación de propuestas que fomentaran el pensamiento crítico de la comunidad.

\section{Marco teórico-prácticos básicos}

La experiencia se enmarca en la educación matemática crítica (EMC), que en términos simplificados es una enseñanza que parta del contexto y cuyo resultado final trascienda, modifique o busque relacionarse con el mismo. Trabajar con la matemática crítica implica entender que lo social va más allá de las interacciones en el aula, busca que los alumnos y profesores sean actores dentro de su propio contexto, que identifiquen problemáticas que directa o indirectamente les afecten y que busquen posibles soluciones a las mismas partiendo de la realidad individual o colectiva en la que estén. En la EMC, la matemática además de ser un cuerpo de conocimientos teóricos y prácticos, se transforma en una herramienta que desarrolla las potencialidades críticas; buscando que lo social penetre en el aula y que a su vez, el aula se integre a lo social en un proceso de retroalimentación. (Valero, 2002)

La educación matemática tradicional sigue el paradigma del ejercicio. Este paradigma contrasta con varios posibles escenarios de investigación que invitan a los estudiantes a involucrarse en un proceso de exploración y explicación. La distinción entre el paradigma del ejercicio y los escenarios de investigación se combina con la distinción entre tres posibles tipos de referencia que proveen significado a los conceptos matemáticos y a las actividades dentro del salón de clase. Así, tenemos referencia a las matemáticas per se, a una semirrealidad y a situaciones de la vida real. Moverse del paradigma del ejercicio hacia los escenarios de investigación puede contribuir a relegar a las autoridades del salón de clase de matemáticas tradicional y, en cambio, resaltar el papel de los estudiantes como sujetos activos de su propio proceso de aprendizaje. En este trabajo, nos movemos de la referencia a las matemáticas per se hacia la referencia a la vida real, porque puede contribuir a ofrecer recursos para la reflexión sobre las matemáticas y sus aplicaciones (Skovsmose, 2000).

En cuanto al aprendizaje geométrico asumimos que el aprendizaje de la geometría se debe interiorizar desde una perspectiva espacial, ya que la geometría es la percepción de la realidad (Alsina, 1997); y utilizamos los niveles del modelo de aprendizaje de la geometría de Van Hiele (año de texto de Van Hiele, citado en Alsina, 1997). 
Nivel básico (1): Visualización

Observación y construcción de modelos de poliedros regulares.

Nivel de análisis (2)

Completación al cuadrado de elementos constituyentes (caras, vértices, aristas).

Nivel de deducción informal (3)

Relación de dualidad

Nivel de deducción (4)

Demostración de $<<$ sólo 5 $>>$

Nivel de rigor (5)

Demostración formal de la existencia efectiva y clases de simetría de los grupos puntuales del espacio.

\section{Descripción general de la experiencia de aula}

Para el desarrollo de la experiencia en el aula, se utilizó una metodología participativa de creación de ambientes. En 4 sesiones de clase se caracterizó la importancia de generar un cambio, y con ayuda de los estudiantes, se planteó una secuencia de actividades que lograra incentivarlos a utilizar el pensamiento crítico y la matemática para analizar problemas de su contexto social. Las sesiones trabajadas con los estudiantes utilizaron materiales audiovisuales y físicos y se plantearon de la siguiente manera:

Actividad 1: representando mi entorno cuyo propósito era la identificación del contexto social y geográfico de los estudiantes y se desarrolló realizando un dibujo de su familia, entorno y lugar de habitación, y posteriormente socializando los dibujos para buscar convergencias.

Actividad 2: "Googleando" mi casa y mi colegio. El propósito de esta actividad era trabajar con mapas virtuales para conocer la relación personal con el contexto geográfico; ésta fue una sesión en un entorno virtual, donde se localizaron los lugares de residencia y se realizó una estimación de qué tan cerca o lejos viven del colegio los estudiantes.
Actividad 3: problematizando $\mathrm{mi}$ entorno. El Propósito en la actividad era seleccionar una problemática para buscar soluciones pertinentes, ya fuera en las instituciones encargadas o propias; retomando los primeros dibujos y la tabla de convergencias se realizó un estudio sobre las problemáticas sociales del entorno en trabajo cooperativo.

\section{Logros y dificultades evidenciadas}

Frente a la experiencia, todos los logros se encaminan al pensamiento crítico de los estudiantes. Con esta propuesta logramos involucrar a los estudiantes en la resolución de problemáticas de su comunidad, además de presentar la matemática como una herramienta útil en el desarrollo de competencias ciudadanas. Cabe resaltar que el PEI del colegio ayudó bastante en este desarrollo pues busca formar ciudadanos reflexivos. No se presentaron mayores dificultades en la experiencia, y todas se refieren a los espacios virtuales para la realización de las actividades.

El logro principal de la experiencia en el aula fue el diseño de una propuesta de enseñanza que lograra responder a las necesidades de la comunidad y con la que se lograra desarrollar competencias críticas en los estudiantes. La experiencia en el aula logró identificar tres grandes problemáticas que afectaban a la comunidad educativa. Las primeras de ellas se refieren a la violencia y la inseguridad que los estudiantes visualizan en su cotidianeidad y frente a las cuales no ven solución por parte de las autoridades competentes. La segunda problemática se refiere a los problemas de movilidad y tránsito, pues al estar cerca de una autopista urbana, el colegio y sus vías aledañas se encuentran inmersos en el tráfico constante y se ve afectado por ello.

\section{Reflexión final y conclusiones}

Destacamos la importancia que tienen propuestas de aula que busquen el mejoramiento día a día de la educación matemática, estas innovaciones no son fáciles de gestar y la realización de las mismas exige compromiso social frente a los estudiantes y la comunidad en general. No podemos olvidar que el 
contexto social del estudiante influye en los procesos de enseñanza aprendizaje y que los enfoques cualitativos son pertinentes para la realización de este tipo de trabajos ya que permite una mejor recolección y análisis de los datos obtenidos, puesto que este tipo de enfoque da profundidad a los datos, la contextualización del ambiente o entorno, los detalles y las experiencias únicas, aportando un punto de vista "fresco, natural y holístico" de los fenómenos

La propuesta por el momento queda planteada, pero es nuestro compromiso y deseo que propuestas como estas sigan implementándose. El desarrollo de competencias críticas en los estudiantes es una solución posible a muchas problemáticas sociales de nuestros estudiantes, $\mathrm{y}$ hace visible las matemáticas más que conocimientos teóricos inertes son herramientas actuales y eficaces para analizar, modelar, razonar, comunicar y plantear soluciones a problemas cotidianos.

\section{Referencias}

Alsina, C. (1997). Invitación a la didáctica de la geometría. Madrid: Sintesis.

Skovsmose. (2000). Escenarios de investigación. Revista EMA, (6) 1 p. 3-26. Rescatado el 6 de Abril de 2012 de http://funes.uniandes.edu. co/1122/1/70_Skovsmose2000Escenarios_ RevEMA.pdf.

Valero, P. (2002). Consideraciones sobre el contexto yla educación matemática para la democracia. 